\title{
Considering on the exercise rehabilitation and the Journal of Exercise Rehabilitation
}

\author{
Chang-Ju Kim (iD https://orcid.org/0000-0003-4749-5795
}

Despite the remarkable progress of medicine and science, we still suffer from incurable diseases. The development of society has resulted in new diseases that did not exist in the past. The economic imbalance between countries or regions is a factor for new social conflicts and related diseases. Excessive nutrition and lack of exercise have led to the expansion of metabolic diseases in modern humans. Therefore, there is an urgent need for alternative and complementary therapies to meet the new disease paradigm that modern science and medicine can't afford.

Exercise is a human hobby and also plays a technical role in games like the Olympics. Exercise is known to have many beneficial effects on human body. The remarkable effects of exercise, such as neurogenesis and antiapoptotic effects, have been recently discovered and researches on the mechanisms of exercise have also made many advances.

Exercise rehabilitation is a part of rehabilitation, and it is also important to act as an adjunct to traditional rehabilitation therapy. However, the efficacy of exercise rehabilitation is very broad and, in some cases, exercise rehabilitation has different therapeutic areas than traditional rehabilitation. Exercise rehabilitation can be presented as a solution to new diseases in modern society. Exercise rehabilitation acts as an alternative strategy to replace traditional medicine in economically challenging areas. Exercise rehabilitation is very effective in overcoming metabolic diseases, which is a modern disease. Exercise rehabilitation is known to be effective for neuropsychiatric disorders, such as depression, and has no side effects. In particular, exercise rehabilitation showed as a new possibility for intractable diseases, such as autism, attention deficit hyperactivity disorder, and schizophrenia etc.

The purpose of the Journal of Exercise Rebabilitation (JER), the journal of the Korean Society of Exercise Rehabilitation, is to inform the effects of exercise rehabilitation on various diseases and to clarify the therapeutic mechanisms of exercise rehabilitation. The JER aims to exist as a medium for objectively and scientifically verifying the efficacy of exercise rehabilitation being performed all over the world.

Through the efforts of the editorial board of the JER, we expect that exercise rehabilitation will become a therapeutic strategy to overcome regional and economic disparities and develop into a new therapeutic field that challenges contemporary incurable diseases.

\section{CONFLICT OF INTEREST}

No potential conflict of interest relevant to this article was reported.

President of the Korean Society of Exercise Rehabilitation Department of Physiology, College of Medicine, Kyung Hee University, 26 Kyungheedae-ro, Dongdaemun-gu, Seoul 02447, Korea E-mail: changju@khu.ac.kr 